Cahiers de philosophie de l'université de

\title{
La crise de l'humanité européenne selon Patočka
}

\author{
Émilie Tardivel
}

\section{(2) OpenEdition}

Journals

Édition électronique

URL : https://journals.openedition.org/cpuc/1151

DOI : 10.4000/cpuc. 1151

ISSN : 2677-6529

Éditeur

Presses universitaires de Caen

\section{Édition imprimée}

Date de publication : 15 décembre 2010

Pagination : 131-144

ISBN : 978-2-84133-365-3

ISSN : 1282-6545

Référence électronique

Émilie Tardivel, «La crise de l'humanité européenne selon Patočka », Cahiers de philosophie de l'université de Caen [En ligne], 47 | 2010, mis en ligne le 02 septembre 2020, consulté le 31 janvier 2023 URL : http://journals.openedition.org/cpuc/1151; DOI : https://doi.org/10.4000/cpuc.1151

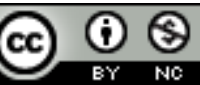

Creative Commons - Attribution - Pas d'Utilisation Commerciale 4.0 International - CC BY-NC 4.0 https://creativecommons.org/licenses/by-nc/4.0/ 


\section{La crise de l'humanité européenne selon Patočka}

Vielleicht ist der Sinn von Europas Untergang positiv. Jan Patočka

«P

EuT-ÊTRe le déclin de l'Europe a-t-il un sens positif » ${ }^{1}$. Loin d'être un hapax dissimulé dans un texte secondaire, cette affirmation traverse au contraire toutes les analyses patočkiennes de la crise européenne, devenue, au moment où il écrit, une crise mondiale. C'est pourquoi il ne faudrait pas trop vite conclure à l'européocentrisme de Patočka. D’emblée, il s'agirait de distinguer deux perspectives: (1) la perspective descriptive, selon laquelle l'histoire mondiale est essentiellement une généralisation de l'histoire européenne; (2) la perspective normative, selon laquelle cette généralisation a moins été jusqu’à présent une généralisation de l’universel que la généralisation d'un particulier que l'on a tenté de faire passer pour l'universel. C'est même à l'aune de cet argument que Patočka vient critiquer l'un de ses pères spirituels: «Husserl lui-même succombe à cette tentation en faisant passer l'idéal de la ratio européenne pour l'entéléchie universelle de l'humanité $»^{2}$. La conséquence inévitable d'une telle approche se trouve dans le sinistre passage de la conférence de 1935, où Husserl exclut de l'humanité européenne et, partant, de l'humanité comme telle, Esquimaux, Indiens et Tsiganes ${ }^{3}$.

1. J. Patočka, «Die nacheuropäische Epoche und ihre geistigen Probleme» [1 ${ }^{\mathrm{re}}$ moitié des années 70], Péče o duši, III, Prague, Archivní soubor, 1988, p. 375; trad. fr. E. Abrams, "L'époque posteuropéenne et ses problèmes spirituels », L'Europe après l'Europe, Lagrasse, Verdier, 2007, p. 212 (traduction modifiée).

2. J. Patočka, «Die Selbstbesinnung Europas» [ $1^{\text {re }}$ moitié des années 70$]$, Perspektiven der Philosophie, Amsterdam - Atlanta, Neues Jahrbuch, 1994, vol. 20, p. 273 ; trad. fr. E. Abrams, «Réflexion sur l'Europe», Liberté et sacrifice, Grenoble, Millon, 1990, p. 212 (traduction modifiée).

3. E. Husserl, «Die Krisis des europäischen Menschentums und die Philosophie» [1935], Die Krisis der europäischen Wissenschaften und die transzendentale Phänomenologie, La Haye, 
Patočka n'a donc aucune nostalgie de l'hégémonie européenne, brisée par la Première Guerre mondiale. Pour autant, il ne nie pas que cette hégémonie passée, qui ne peut se justifier à partir d'une prétendue supériorité de la civilisation européenne sur les autres civilisations, s'explique néanmoins à partir d'une certaine spécificité européenne. Certes, la relecture patočkienne de l'histoire européenne, à l'instar de la relecture husserlienne, est commandée par l'exigence de revenir à cette spécificité pour comprendre le déclin de l'Europe, c'est-à-dire pour comprendre ce qui a été dévoyé, à quel moment et de quelle manière. Mais, contrairement à Husserl, Patočka n'a jamais considéré qu'un simple retour à cette spécificité puisse sortir l'Europe de la crise. L'Europe ne doit pas simplement revenir à elle mais s'ouvrir à l'autre, ou plutôt revenir à elle depuis l'autre:

La généralisation de l'Europe [coïncidant avec la fin de son hégémonie] doit nécessairement conduire à un débat et, partant, à une revalorisation des traditions culturelles considérées comme mortes et insignifiantes, mais qui fournissent à la pensée européenne [europäischen Gedanken] une assise factuelle absolument nécessaire ${ }^{4}$.

Autrement dit, le déclin de l'Europe a un sens positif, dans la mesure où il ouvre la possibilité non d'un simple retour mais d'un retour critique à l'idéal de la ratio, ou d'un approfondissement de la ratio européenne en ratio mondiale, donc en ratio tout court. Par conséquent, il s'agirait de voir dans la mondialisation cette possibilité d'approfondissement, ou encore d'Aufhebung, où serait supprimé le particulier et conservé ce qu'il $y$ a d'authentiquement universel en Europe. Il y aurait ainsi une chance de salut dans le naufrage même de l'Europe, à l'image du rhinocéros dans $E$ la nave va, qui devient, à la fin du film de Fellini, le symbole d'une renaissance possible de l'Europe à partir d'un ressortissant de ces «ménageries foraines [Jahrmarktsmenagerien] » européennes qu'évoque Husserl à propos des Esquimaux et des Indiens (le journaliste italien Orlando, qui réchappe du naufrage avec le rhinocéros, jusque-là considéré comme un poids mort, n'annonce-t-il pas une «grande nouvelle»: «Saviez-vous

Nijhoff (Husserliana; 6), 1954, p. 318; trad. fr. G. Granel, «La crise de l'humanité européenne et la philosophie ", La crise des sciences européennes et la phénoménologie transcendantale, Paris, Gallimard, 1999, p. 352. Voir le commentaire qu'en fait Derrida dans une longue note: De l'esprit, Paris, Galilée, 1987, p. 94-96. Voir aussi les développements de Jean-Luc Marion sur la définition de l'homme par le je transcendantal comme "proscription»: Certitudes négatives, Paris, Grasset, 2010, chap. I, $\$ 5$.

4. J. Patočka, «Die Selbstbesinnung Europas», p. 273; trad. fr. E. Abrams, «Réflexion sur l'Europe», p. 212 (traduction modifiée). 
que le rhinocéros donne un excellent lait?»). Reste à savoir comment Patočka pense cette Aufhebung de la ratio européenne. C'est la question à laquelle nous tenterons de répondre à partir d'une relecture historique et systématique de ses textes sur l'Europe, des années trente aux années soixante-dix.

\section{La genèse de la question de l'Europe}

\section{La double filiation et l'écart}

Patočka inaugure sa réflexion sur l'Europe en s'inscrivant d'emblée dans une double filiation, tchèque et allemande, tout en se positionnant à l'égard de ses illustres prédécesseurs. Le premier texte qu'il consacre à cette question s'intitule en effet: «La conception de la crise spirituelle de l'humanité européenne chez Masaryk et chez Husserl». Il l'écrit en 1936, à peine un an après deux événements: la démission de Masaryk de la présidence tchèque et le cycle de conférences sur la crise de l'humanité européenne, données par Husserl à Prague, en novembre 1935, sur invitation du secrétaire tchèque du Cercle philosophique, qui n'était autre que Patočka. C'est à cette même époque qu'il reçoit de Husserl le lutrin que ce dernier avait reçu de Masaryk à Leipzig. Devenant par ce geste l'héritier d'une grande tradition, comme il l'avouera lui-mêmes, il assume donc la tâche qui lui incombe dans un texte où son premier mouvement consiste à ramener les deux pensées dont il hérite à leur dénominateur commun : «[...] la conviction que l'humanité européenne traverse une crise spirituelle prolongée, dont les racines remontent aux débuts mêmes de la pensée moderne». Autrement dit, indépendamment de la manière dont ils interprètent les symptômes et le remède de la crise, les deux élèves de Brentano sont d'accord pour en voir l'origine dans la pensée moderne. Or que signifie cette formule de "pensée moderne» ? Pour résumer l'idée que développe Patočka dans le texte de 1936, nous dirions que la pensée moderne est une pensée nécessairement confrontée au problème de la corrélation, c'est-à-dire à la difficulté de comprendre comment l'homme peut être à la fois une condition (pour autant qu'il constitue le monde sur le fondement de l'évidence inébranlable du cogito) et un moment du monde (pour autant qu'il est lui-même une partie de ce qu'il constitue). Or, «dans sa perplexité, rappelle Patočka, la philosophie moderne cherche refuge dans l'idée de concevoir l'homme

5. J. Patočka, «Erinnerungen an Husserl» [1976], Die Welt des Menschen - Die Welt der Philosophie: Festschrift für Jan Patočka, W. Biemel (éd.), La Haye, Nijhoff, 1976, p. XV. 
comme une partie de la nature, envisagée, bien sûr, de façon moderne, c'est-à-dire more geometrico». Par conséquent, poursuit-il,

l'homme aussi devient, pour elle, géométrique, il perd la liberté pour la vérité [svobodu k pravdè], il ne vit plus au milieu des choses telles qu'elles sont, mais au milieu de ses processus subjectifs, qu'aucun pont ne relie à la réalité vraie [pravé skutečnosti] ${ }^{6}$.

La pensée moderne ne substitue donc pas seulement aux choses les résultats des opérations subjectives de l'homme, les résultats de l'objectivation des choses, elle substitue aussi à l'homme le résultat de l'objectivation de l'homme. Cela revient à soutenir que la pensée moderne résout moins qu'elle n'accroît le problème auquel elle est nécessairement confrontée, en opérant une sorte de disjonction entre l'homme et le monde, d'autant plus radicale qu'elle ne passe pas uniquement entre l'homme et les choses, mais traverse l'homme lui-même.

Comment réconcilier l'homme et le monde, c'est-à-dire l'homme avec lui-même? C'est dans la réponse à cette question que Patočka marque son double écart. D’un côté, il reproche à Masaryk de sacrifier son analyse philosophique sur l'autel d'une critique théologique naïve de la pensée moderne. Bien entendu, cela ne veut pas dire que toute critique théologique soit nécessairement «naïve». C'est justement sur la question de la foi que Patočka vient se démarquer de Husserl. Il vient précisément se démarquer de son intellectualisme, Husserl considérant la foi comme une "position théorique ", alors qu'elle relève, pour Patočka, d' «[...] une décision personnelle, qui ne se fonde pas sur des arguments, ni ne procède d'aucune théorie, mais plutôt nous y conduit et s'explicite dans des vues spécifiques sur la totalité du monde». Autrement dit, la naïveté de la critique masarykienne ne réside nullement dans son caractère théologique. Elle réside dans sa prétention à juger de la pertinence d'une pensée ailleurs que sur son propre terrain, en l'occurrence, le terrain philosophique. Bref, jugeant philosophiquement irrecevable la critique masarykienne selon laquelle la pensée moderne serait l'expression de l'orgueil titanesque de l'homme moderne, Patočka se rapproche de la solution husserlienne d'une critique de la pensée moderne sur son propre terrain. Il voit néanmoins la justesse de l'idée masarykienne de la foi, en tant que «sentiment de confiance et

6. J. Patočka, «Masarykovo a Husserlovo pojetí duševní krize evropského lidstva» [1936], Češi, I, Prague, Oikoymenh, 2006, respectivement p. 22 et 28 ; trad. fr. E. Abrams, «La conception de la crise spirituelle de l'humanité européenne chez Masaryk et chez Husserl», La crise du sens, I, Bruxelles, Ousia, 1985, respectivement p. 20 et 30 (traduction modifiée). 
d'amour dans le don de soi au monde [odevzdanosti světu] » ${ }^{7}$, dont s'inspirera sans doute l'idée de la percée en tant que mouvement de la conquête de soi par le don de soi au monde ${ }^{8}$. Cette idée doit être cependant libérée du rationalisme théologique qui la sous-tend, pour être réenvisagée sur le terrain de la pensée moderne, dans la perspective radicalement subjective ouverte par Descartes et renouvelée par Husserl. De manière totalement conséquente, Patočka conclut le texte de 1936 en soutenant que la crise de l'humanité européenne ne peut être résolue sans que soit posée «la question de la foi personnelle sur le terrain du subjectivisme radical». En d'autres termes, il s'agit de poser, sur le terrain husserlien, la question masarykienne de cette décision qui consiste à se laisser revendiquer par le monde pour manifester la vérité du monde. À travers cette conclusion, nous constatons que l'écart de Patočka s'accomplit dans la relève de sa double filiation, dans la mesure où la phénoménologie, dont la paternité revient à Husserl, aurait le pouvoir de sortir l'Europe de la crise, en venant poser, outre la question husserlienne de la raison, la question masarykienne de la foi. Mais au lieu de la "question de la foi", nous pourrions anticiper en parlant de la «question de la liberté», puisque la liberté, qui s'accomplit dans le mouvement de percée, deviendra le terme patočkien pour la foi en tant que "don de soi au monde et aux tâches qui nous incombent" ${ }^{9}$. Ainsi s'esquissent, dès le milieu des années trente, les choix fondamentaux de Patočka sur la question de l'Europe.

\section{Le concept de surcivilisation}

Environ vingt ans séparent cette première étude des développements ultérieurs de Patočka sur la question de l'Europe. Ce silence pourrait sans doute s'expliquer par une prise de conscience qui se produit en 1941, sur fond des événements tragiques qui ravagent la Tchécoslovaquie (avec l'annexion des Sudètes en 1938, l'établissement du protectorat de Bohême-Moravie en 1939, etc.), l'Europe et le monde: "C'est l'Europe, déclare Patočka dans un texte extrêmement court sur la ratio européenne, qui est en elle-même [v sobě]

7. Ibid., respectivement p. 32 et 26 ; trad. fr. respectivement p. 36 et 26 (traduction modifiée).

8. J. Patočka, «K prehistorii vědy o pohybu : svět, země, nebe a pohyb lidského života » [1965], Přirozený svět a pohyb lidské existence, I, Prague, Archivní soubor, 1980, p. 1.2.9-1.2.12 ; trad. fr. E. Abrams, «Notes sur la préhistoire de la science du mouvement: le monde, la terre, le ciel et le mouvement de la vie humaine", Le monde naturel et le mouvement de l'existence humaine, Dordrecht, Kluwer, 1988, p. 10-12.

9. J. Patočka, «Masarykovo a Husserlovo pojetí duševní krize evropského lidstva», p. 33 et 26 ; trad. fr. E. Abrams, «La conception de la crise spirituelle... », p. 37 et 26 (traduction modifiée). 
dépourvue de clarté et de certitude ${ }^{10}$. Au début des années quarante, Patočka réaffirme donc son désaccord avec l'intellectualisme husserlien: l'Europe ne sortira pas de la crise en apportant une solution au problème du fondement commun de la philosophie et de la science, dans la mesure où la crise, plus profonde qu'un simple conflit d'intentionnalités, relève de l'essence même de l'Europe. En pleine Seconde Guerre mondiale, Patočka vient ainsi expérimenter l'essence tragique de l'Europe, expérience qui donnera lieu à cette grande thèse des années soixante-dix, selon laquelle le principe du déclin est essentiellement identique au principe de l'essor et de la genèse de l'Europe, et donc à l'idée selon laquelle la crise est déjà contenue dans l'héritage grec. Mais avant les années soixante-dix, c'est au début des années cinquante qu'il commence à tirer les leçons de cette expérience, dans un texte qui s'intitule: «La surcivilisation et son conflit interne». Par le concept de «surcivilisation [nadcivilizace]», Patočka ne désigne pas une civilisation qui serait «supérieure» aux autres civilisations, mais une civilisation qui s'efforce de pallier son manque d'universalité par un processus de rationalisation qu'elle étend à tous les domaines (économique, politique, spirituel). Bien entendu, l'Europe correspond parfaitement à ce concept, dont Patočka, pour l'instant, fait remonter le principe au XVI siècle, plus précisément, à une distinction métaphysique qui vient rendre possible la définition moderne de la substance (ce que nous tenons pour certain, disponible, calculable $)^{11}$. Et c'est ce concept de surcivilisation qui, né d'une crise au XVI ${ }^{\mathrm{e}}$ siècle, est lui-même en crise au $\mathrm{XX}^{\mathrm{e}}$ siècle, crise qui s'atteste, au moment de la Guerre froide, dans l'opposition entre une version modérée et une version radicale de la surcivilisation ${ }^{12}$, opposition qui redouble le conflit interne à la civilisation européenne entre une version spirituelle (avant le $\mathrm{XVI}^{\mathrm{e}}$ siècle) et une version matérielle de la civilisation (après le $\mathrm{XVI}^{\mathrm{e}}$ siècle). Or l'opposition entre les deux versions de la surcivilisation réside dans le rapport au passé: alors que la version modérée est ouverte au passé sur la question des fins, la version radicale étend à la question des fins le même processus de rationalisation qu'à la question des moyens. La surcivilisation radicale serait ainsi le symptôme d'une crise du second

10. J. Patočka, «Evropský rozum» [1941], Péče o duši, I, Prague, Oikoymenh, 1996, p. 116; trad. fr. E. Abrams, «La raison européenne», L'Europe après l'Europe, p. 187 (nous soulignons).

11. Nous subodorons que Patočka se réfère ici à la définition du concept objectif d'étant chez Suárez et la seconde scolastique. Voir sur ce point: J.-F. Courtine, Suárez et le système de la métaphysique, Paris, PUF, 1990.

12. J. Patočka, «Nadcivilizace a její vnitřní konflikt» [1 ${ }^{\mathrm{re}}$ moitié des années 50], Péče o duši, $\mathrm{I}$, p. 250 ; trad. fr. E. Abrams, «La surcivilisation et son conflit interne», Liberté et sacrifice, p. 109. 
degré, exprimant la négation $d u$ monde au profit d'un monde dans lequel l'homme ne vit plus qu' "au milieu de ses processus subjectifs », c'est-à-dire du monde de l'idéologie.

Pour sauver l'Europe de la crise paroxystique qui l'oppose à elle-même à l'échelle du monde, Patočka propose une solution qui nous interpelle audelà du contexte de la Guerre froide. Si l'exigence phénoménologique d'un retour au monde comme tel suppose un refus de l'idéologie, cela ne veut pas dire qu'il faille pour autant épouser aveuglément la version modérée, c'est-à-dire libérale.

La crise du libéralisme, comme plus généralement celle de la civilisation modérée, tient peut-être au fait que la ratio, en tant qu'élément de la vie et de la diffusion de cette forme de civilisation, n'est pas l'élément de la décision ultime $[\ldots]$.

Au début des années cinquante, Patočka reprend donc la question de la décision, la question de la foi, pour critiquer dans la version libérale, ou modérée, l'idée qui consiste à penser que la fides est essentiellement reconductible à la ratio. Or, dans la pensée de Patočka, il est vrai que la foi ne peut être reconduite ni à la raison en tant qu'étant subjectif, ni à Dieu en tant qu'étant objectif, ni à un quelconque étant. L'expérience de la foi n'est que l'autre nom, le nom «non phénoménologique», de l'expérience de la liberté, dont l'essai de 1953, Le platonisme négatif, montre avec force qu'elle est l'expérience d'une transcendance négative, d'une transcendance sans transcendant ${ }^{13}$. L'expérience de la liberté consiste en un dépassement de l'étant mondain, qui ne conduit jamais à un étant absolu, mais ouvre à l'Idée, entendue au sens heideggérien, voire déjà patočkien, c'est-à-dire au monde comme tel. Précisons que, si l'expérience patočkienne de la liberté est l'expérience d'une transcendance négative, elle est toutefois l'expérience d'une liberté «positive», car elle est l'expérience d'une liberté "pour» l'Idée. Dans un premier temps, c'est ce qui la différencie de l'expérience libérale de la liberté, qui est l'expérience d'une liberté négative: «[...] la liberté est présente [dans la version libérale ou modérée], bien qu'on ne voie pas clairement "pour" quoi cette liberté est liberté». Dans un second temps, ce qui la différencie de l'expérience libérale de la liberté, c'est qu'elle est «négative» en un tout autre sens. Contrairement à l'expérience libérale, l'expérience patočkienne de la liberté n'est pas une expérience dont l'homme est à l'initiative. Dans l'expérience patočkienne de la liberté, l'homme répond à l'appel de l'Idée. Plus précisément, il répond à l'Idée en tant qu'appel,

13. J. Patočka, «Negativní platonismus» [1953], Péče o duši, I, p. 303-336; trad. fr. E. Abrams, "Le platonisme négatif», Liberté et sacrifice, p. 53-98. 
puisque Patočka définit l'Idée comme "pur appel de la transcendance» ${ }^{14}$. Autrement dit, contrairement à l'expérience libérale de la liberté, expérience positive d'une liberté négative, l'expérience patočkienne doit être vue comme l'expérience négative d'une liberté positive, au sens où elle est l'expérience d'une liberté «par» et "pour» l'Idée. Ne désigne-t-elle pas dès lors cette foi convertie en une expérience dans laquelle l'Europe est appelée à transcender le monde de l'idéologie vers le monde de l'Idée, qui n'est pas un arrière-monde mais le monde comme tel? «La solution, s'interroge Patočka, résiderait-elle dans la conversion [obratu] du positif en négatif de l'élément non rationnel que nous venons d'évoquer en passant ${ }^{15}$ ?»

\section{L'Aufhebung de la ratio européenne}

\section{Les Grecs et le soin de l'âme}

De nouveau, environ vingt ans séparent cette seconde étude des développements ultérieurs de Patočka sur la question de l'Europe. Mais, cette fois-ci, le silence est moins à mettre sur le compte de la situation internationale que sur celui de la situation intellectuelle du philosophe tchèque. L'essai de 1953, Le platonisme négatif, représente en effet un moment charnière, dans la mesure où il amorce le virage vers la constitution, au cours des années soixante, du système phénoménologique patočkien. Nous ne pouvons restituer, dans le cadre du présent article, cette constitution. Notons simplement que Patočka sera presque totalement accaparé par ce travail, qui lui permettra, au début des années soixante-dix, de revenir à la question de l'Europe avec un dispositif théorique bien mieux ajusté à la complexité de l'entreprise. C'est au début de "L'Europe après l'Europe», manuscrit allemand des années soixante-dix, qu'il formule cette entreprise avec le plus de précision: «Faire tourner toute la marche de l'histoire européenne autour d'un seul principe $[\ldots] »^{16}$, auquel il serait dès lors possible de reconduire la genèse, l'essor et le déclin de l'Europe. Or quel est ce principe? Pour le découvrir, Patočka va tout d'abord opérer une réduction phénoménologique de l'Europe au thème qui la fonde. Dans le séminaire tchèque de 1973, qui a pour titre Platon et l'Europe, il vient ainsi montrer que le «soin de l'âme

14. J. Patočka, «Negativní platonismus»,p. 333; trad. fr. E. Abrams, «Le platonisme négatif», p. 95.

15. J. Patočka, «Nadcivilizace a její vnitřní konflikt», respectivement p. 285, 261 et 286; trad. fr. E. Abrams, «La surcivilisation... », respectivement p. 154-155, 123 et 155 (traduction modifiée).

16. J. Patočka, «Europa und Nach-Europa» [1 $1^{\text {re }}$ moitié des années 70], Ketzerische Essais zur Philosophie der Geschichte, Stuttgart, Klett - Cotta, 1988, p. 207; trad. fr. E. Abrams, "L'Europe et après», L'Europe après l'Europe, p. 37. 
[péče o duši] », plus précisément, l'exigence de «prendre soin de l'âme [ $\tau \tilde{\eta} \varsigma$

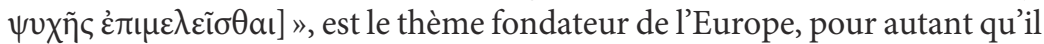
constitue le thème que partagent tous les philosophes grecs au-delà de la conception qu'ils ont de l'âme: «[...] tous, explique Patočka, aussi bien les tenants de l'âme immortelle que ceux qui posent une âme mortelle et corruptible, tous affirment qu'il faut se soucier de l'âme $[\ldots] »^{17}$. Le soin de l'âme serait par là même le principe spirituel de la genèse de l'Europe. Or que signifie exactement "soin de l'âme", et que signifie donc ici "principe spirituel »? Pour comprendre ce que le "soin de l'âme» recouvre, Patočka va procéder en trois temps: (1) il va d'abord analyser le soin de l'âme chez Démocrite et chez Platon, dont l'antagonisme (entre la doctrine démocritéenne des atomes, à l'origine du matérialisme, et la doctrine platonicienne des idées, à l'origine de l'idéalisme) révèle par contraste un accord profond sur ce principe dans la philosophie grecque; (2) il va ensuite approfondir l'analyse du soin de l'âme platonicien, en mettant en évidence son caractère décisif et systématique à travers sa partition en trois grands projets: ontocosmologique, politique et existentiel; (3) il va enfin mettre en évidence l'apport essentiel d'Aristote au soin de l'âme platonicien. Démocrite, Platon, Aristote: voici le mouvement qu'accomplit Patočka dans sa réflexion sur le soin de l'âme chez les philosophes grecs. De cette grande et intense réflexion, nous ne donnerons ici que les principales conclusions.

La première idée consiste à poser que, sur le problème du soin de l'âme, la période socratique rompt moins avec la période présocratique qu'elle n'en reprend l'héritage. Or, comme l'écrit Patočka, cette reprise est à comprendre au double sens de l'Aufhebung, de la suppression et de la conservation. De la période présocratique représentée par Démocrite, Platon conserve au

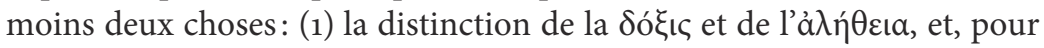

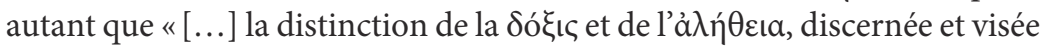
dans la connaissance des choses, est à l'origine de la distinction morale ${ }^{18}$, du mal et du bien; (2) l'âme comme «ce qui en l'homme est capable de vérité $»^{19}$, c'est-à-dire comme ce qui en l'homme est capable de voir le primat

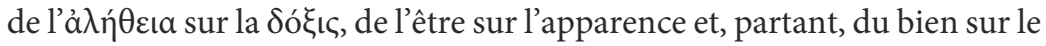
mal. Chez Platon, comme chez Démocrite, prendre soin de l'âme signifie donc prendre soin de ce qui permet à l'homme de se rapporter explicitement à l'à $\lambda \eta ́ \theta \varepsilon ı$, à l'être et, partant, au bien. Malgré l'affinité profonde qui

17. J. Patočka, «Platón a Evropa» [1973], Péče o duši, II, Prague, Oikoymenh, 1999, p. 16o; trad. fr. E. Abrams, «Platon et l'Europe», Platon et l'Europe, Lagrasse, Verdier, 1997, p. 20.

18. J. Patočka, «Démokritos a Platón jako zakladatelé metafyziky» [ $1^{\text {re }}$ moitié des années 70], Péče o duši, II, p. 359; trad. fr. E. Abrams, «Démocrite et Platon, fondateurs de la métaphysique», Platon et l'Europe, p. 268.

19. J. Patočka, «Platón a Evropa», p. 172; trad. fr. E. Abrams, «Platon et l'Europe», p. 35. 
unit ces deux démarches, le soin de l'âme platonicien marque toutefois une rupture. Patočka parle bien d' "Aufhebung», donc non seulement de conservation, mais aussi de suppression. Or, ce que Platon supprime de la démarche démocritéenne, c'est précisément l'idée selon laquelle le soin de l'âme serait un moyen en vue du savoir. Pour Platon, c'est à l'inverse le savoir qui est un moyen en vue du soin de l'âme. Le savoir n'est plus envisagé comme la fin mais, au contraire, comme l'«organon d'une activité originaire, d'une praxis ${ }^{20}$. La seconde idée consiste donc à poser que la démarche platonicienne constitue une avancée décisive, dans la mesure où elle redéfinit le soin de l'âme comme une activité qui n'a d'autre fin qu'elle-même («une praxis»), plus précisément, qui n'a d'autre fin que le

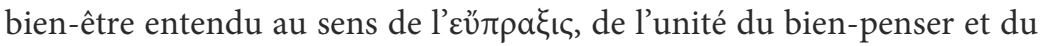
bien-agir. Or cette avancée s'atteste avant tout dans la possibilité d'une investigation désormais systématique du monde. Avec Platon, le soin de l'âme se divise maintenant en trois grands projets: (1) «une doctrine philosophique universelle qui rapporte l'âme à la structure de l'être »; (2) «une doctrine de la vie du philosophe dans la société et dans l'histoire»; (3) «une doctrine de l'âme comme principe de la vie individuelle ${ }^{21}$. La question reste alors de savoir comment Aristote vient apporter sa pierre à l'édifice platonicien. Pour résumer l'argument de Patočka, nous dirions que son apport essentiel réside dans la problématisation du bien vers lequel l'âme tend: «Le bien n'est donc pas quelque élément commun dépendant d'une Idée unique ${ }^{22}$. La troisième idée consiste donc à poser qu'Aristote vient dévoiler la problématicité de la vie à partir d'une critique de l'Idée, qui n'est peut-être pas encore assez radicale, mais qui ouvre toutefois la voie à une indispensable Aufhebung du soin de l'âme platonicien.

\section{Souci d'être ou souci d'avoir}

Pour indispensable qu'elle soit, cette Aufhebung du soin de l'âme platonicien n'aura pas lieu, du moins pas dans l'ordre de la pensée jusqu'à Husserl inclus, et encore moins dans l'ordre politique. Or, ce qui va d'abord intéresser Patočka, c'est justement la façon dont le soin de l'âme platonicien va se réaliser dans l'ordre politique européen, ou plutôt va réaliser l'ordre politique européen. Il n'est en effet possible de parler de l'Europe qu'à

20. J. Patočka, «Europa und Nach-Europa», p. 256; trad. fr. E. Abrams, «L'Europe et après», p. 96 (nous soulignons et modifions la traduction).

21. J. Patočka, «Platón a Evropa», respectivement p. 314, 233 et 314; trad. fr. E. Abrams, «Platon et l'Europe», respectivement p. 193, 105 et 193 (traduction modifiée).

22. Aristote, Éthique à Nicomaque, 1096 b 25. 
partir du moment où elle émerge en tant que réalité politique, c'est-à-dire à partir du Moyen Âge. Pour prouver que le soin de l'âme platonicien est bien le principe spirituel de la genèse de l'Europe, Patočka va donc mettre en évidence le lien entre le projet politique du soin de l'âme platonicien et l'idée du sacrum imperium qui sous-tend l'ordre politique de l'Europe médiévale. Le sacrum imperium européen (par opposition à la version islamique ou encore byzantine) repose sur l'idée de la distinction du pouvoir spirituel et du pouvoir temporel, entendue au sens d'une subordination du pouvoir temporel au pouvoir spirituel. Or c'est cette priorité du pouvoir spirituel sur le pouvoir temporel qui rend assez ambivalent le sacrum imperium européen. D'un côté, il marquerait l'échec du projet politique du soin de l'âme platonicien, pour autant que celui-ci consiste à fonder une communauté autonome par rapport au pouvoir religieux, entendu au

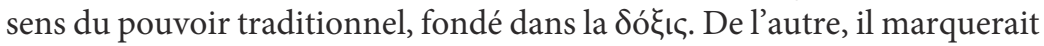
au contraire une reprise de ce projet, pour autant que le pouvoir religieux dont il est ici question constitue désormais un pouvoir proprement spirituel, c'est-à-dire fondé dans l'ả $\lambda \hat{\theta} \theta \varepsilon ı$. Autrement dit, bien qu'il ne puisse pas être conçu comme un parfait accomplissement du projet politique du soin de l'âme platonicien, le sacrum imperium européen perpétue néanmoins le "projet d'un État fondé dans l'esprit ${ }^{23}$ sous une nouvelle forme. Comme l'écrit Patočka, il ne s'agit plus de l'«État temporel des Césars, avec son oscillation trop humaine entre l'arbitraire et la volonté de légalité, entre le despotisme de l'état de nature et le "droit naturel" ", mais d'une "[...] communauté directement fondée dans une vérité absolue, qui n'est pas de ce monde-ci, mais de ce monde-là [...] ${ }^{24}$. Le sacrum imperium européen vient donc moins marquer l'échec du projet politique du soin de l'âme platonicien que celui de son accomplissement par la $\pi$ ó $\lambda$ เ athénienne et l'Empire romain. Née sur les ruines d'Athènes et de Rome, l'Europe vient néanmoins marquer une reprise de leur héritage, c'est-à-dire une reprise du soin de l'âme platonicien. Pour être plus précis, il faudrait ajouter que l'héritage de l'Europe recouvre un double accomplissement du soin de l'âme platonicien: la naissance du politique (Athènes) et la naissance du juridique (Rome). Le politique et le juridique sont dès lors à comprendre comme le déploiement d'un même principe spirituel, comme autant de

23. J. Patočka, «Europa und Nach-Europa», p. 265; trad. fr. E. Abrams, «L'Europe et après», p. 108.

24. J. Patočka, «Evropa a evropské dědictví do konce 19. století» [1975], Péče o duši, III, Prague, Oikoymenh, 2002, p. 86-87; trad. fr. E. Abrams, «L'Europe et l'héritage européen jusqu’à la fin du XIX siècle», Essais hérétiques sur la philosophie de l'histoire, Lagrasse, Verdier, 1999, p. 109 (traduction modifiée). 
jalons dans le mouvement non nécessaire de réalisation d'un monde de la vérité et de la justice, c'est-à-dire du monde comme tel.

Parler de mouvement "non nécessaire», cela revient à poser que le soin de l'âme platonicien, en tant que "principe spirituel ", n'est nullement une «cause». Pour Patočka, il ne s'agit pas en effet de confirmer la thèse de l'idéalisme hégélien selon laquelle l'histoire est essentiellement déterminée par l'esprit. Certes, le soin de l'âme platonicien est un principe spirituel, mais il ne faut pas l'entendre au sens de Hegel. Il faut l'entendre au sens du $\$ 82$ de Sein und Zeit, où Heidegger vient dévoiler que l'histoire n’est pas essentiellement déterminée par un esprit «tombant» dans le temps mais qu'elle se détermine essentiellement en "retombant» de l' «esprit» [ "Geist»], c'est-à-dire du phénomène de la temporalité authentique. Chez Patočka, il s'agit moins de la temporalité authentique que du monde de la vie authentique, mais l'idée reste la même: le principe spirituel n'est en aucun cas une cause, un principe qui détermine l'histoire, mais un idéal, un principe par rapport auquel l'histoire se détermine. Or, après avoir étudié le rôle de l'héritage grec et de l'héritage romain (ou encore de l'hellénisation de l'héritage judéo-chrétien) dans la genèse de l'Europe, Patočka vient montrer que l'histoire européenne connaît un tournant au XVI ${ }^{\mathrm{e}}$ siècle, lié justement, non à la perte, mais à la transformation de l'idéal du soin de l'âme platonicien. L'histoire européenne, qui s'était jusqu'à présent déterminée par rapport au soin de l'âme platonicien en tant que souci d'être, souci de vérité et de justice (de façon extrêmement imparfaite, Patočka en convient), va désormais se déterminer par rapport au souci d'avoir, au souci de domination. Or la grande force de l'analyse de Patočka, c'est d'avoir révélé que cette transformation, qui marque à la fois l'essor et le déclin de l'Europe moderne, ne relève pas du passage d'un premier à un second principe, mais de la radicalisation d'une tendance contenue dans le premier principe. Dans «L'Europe après l'Europe », Patočka soutient explicitement que l'idéal du soin de l'âme platonicien, qui n'est autre que l'idéal de la ratio européenne, est traversé par une tension entre les deux tâches qui le caractérisent: d'une part, l'objectivité de la connaissance, d'autre part, la responsabilité de l'action. Autrement dit, le souci d'avoir n'est nullement un second principe, mais la forme que prend le soin de l'âme platonicien quand, en lui, l'action se subordonne à l'objectivité d'une connaissance par essence non responsable. L'œuvre de Patočka dans son ensemble pourrait être envisagée comme une tentative pour couper court à cette subordination (qui n'est autre que le phénomène de l'idéologie), sans que la connaissance délaisse pour autant le domaine de l'action éthico-politique. Or comment couper court à cette subordination? D'où vient-elle? À travers son essai de platonisme négatif, ou à travers l'apport aristotélicien au soin de l'âme 
platonicien, nous avons déjà entrevu la réponse proposée par Patočka: la possibilité de la subordination de l'action à la connaissance vient de la définition positive de l'Idée (donc aussi de l'idée de l'homme) comme un étant indivisible et immobile, saisissable dans un regard et exprimable dans un discours. Une véritable Aufhebung du soin de l'âme platonicien - ou de la ratio européenne - consiste par conséquent en une critique radicale de l'Idée en son essence métaphysique, comme de ses multiples avatars philosophiques et politiques. Or n'est-ce pas là le travail que Patočka entreprend dès le début des années cinquante, dans le prolongement des efforts de Heidegger?

Comme nous l'aurons compris, la crise de l'humanité européenne est moins pour Patočka une crise scientifique qu'une crise politique, une crise de la vocation de l'Europe à réaliser dans le monde un monde de la vérité et de la justice. Or, bien qu'il fasse remonter le début de la crise au XVI ${ }^{\mathrm{e}}$ siècle, Patočka n'a pour autant aucune nostalgie de l'Europe médiévale, puisqu'il soutient que la fin du Saint-Empire, comme celle de la cité athénienne et de l'Empire romain, a un sens positif. Dans le séminaire tchèque de 1973, nous lisons en effet: «[...] cette désunion [la division des États modernes qui suit l'effondrement du Saint-Empire, au tout début du XIX ${ }^{\mathrm{e}}$ siècle] n'est pas non plus un facteur purement et simplement négatif. Elle signifie l'absence d'une autorité supérieure, unificatrice $[\ldots] »^{25}$. En d'autres termes, Patočka n'a de nostalgie ni de l'hégémonie de l'Europe moderne (comme nous le soulignions en introduction), ni de l'Europe médiévale ou de ses origines antiques. Il nous propose en ce sens une relecture foncièrement optimiste de l'histoire européenne et de sa généralisation en histoire mondiale. Mais il faut bien voir que cet optimisme ne repose absolument pas sur la croyance en une quelconque nécessité historique. Au contraire, il est gagé sur la croyance en cette capacité de l'humanité à tirer les leçons de l'histoire, voire plus fondamentalement à comprendre ce qu'est l' " histoire [Geschichte]». Le déclin de l'Europe n'a de sens positif que parce qu'il invite l'humanité à cette compréhension plus profonde de ce qu'est l' "histoire» - compréhension dont l'Europe a hérité, mais qu'elle a également refoulée, en préférant au souci d'être, souci proprement historique, le souci d'avoir, souci proprement économique. C'est pourquoi l'Europe ne sortira pas de la crise en apportant une solution au problème du fondement commun de la philosophie et de la science, mais en répondant à la question de l'origine commune de la philosophie et de la politique, c'est-à-dire à la question de

25. J. Patočka, «Platón a Evropa», p. 157; trad. fr. E. Abrams, «Platon et l'Europe», p. 17. 
l'«histoire», à la question de «ce qui en l'homme dépasse la sphère de la simple subsistance ${ }^{26}$. Bref, elle ne sortira de la crise qu'en répondant à la question de l'«ek-sistance», ou encore, pour reprendre les concepts de Patočka, à la question de l'«expérience de la liberté [zkušenost svobody]». Avec la généralisation de l'histoire européenne, l'humanité européenne n'a cependant pas le monopole de cette tâche. Comme l'explique Patočka avec lucidité: «Les peuples extra-européens semblent tous avides de s'approprier cet esprit [l'esprit européen] dans l'espoir d'y trouver un secours dans l'indigence, les privations et les maux dont ils souffrent ». Et de s'interroger: «Pourront-ils en accepter les bienfaits sans tomber victimes d'une misère pire encore, aboutissant à la répression massive et à la destruction d'une vie vidée par le souci exclusif des moyens de l'entretenir? ${ }^{27}$.

Émilie TARdivel

26. J. Patočka, «Die Epochen der Geschichte» [1976], Ketzerische Essais zur Philosophie der Geschichte, p. 194-195; trad. fr. E. Abrams, «Le schéma de l'histoire», L'Europe après l'Europe, p. 27 (nous soulignons).

27. J. Patočka, «Die Selbstbesinnung Europas», p. 272 ; trad. fr. E. Abrams, «Réflexion sur l'Europe», p. 211. 